\title{
Opening the black box of design computation
}

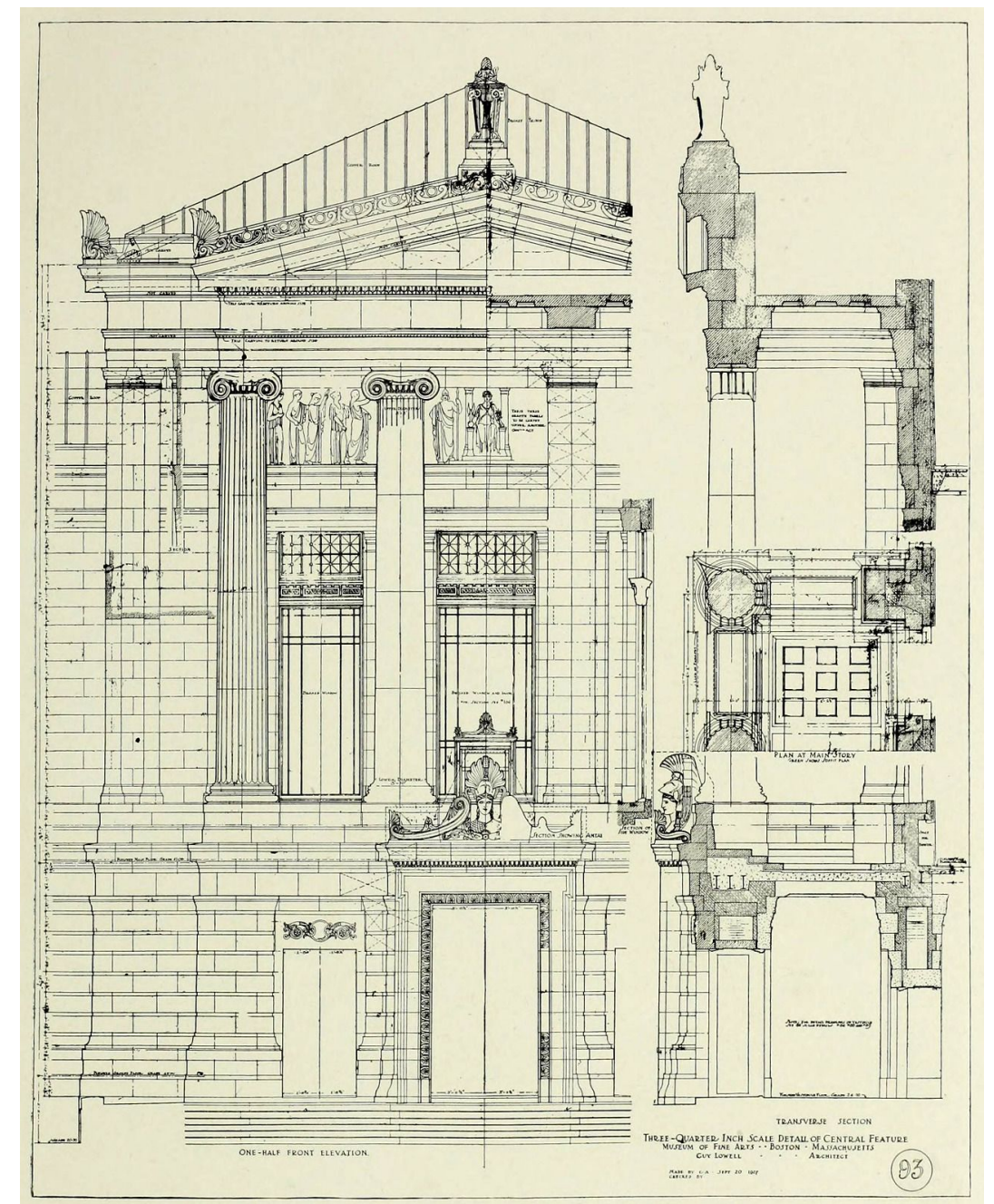

Fig. 1. Construction plan details for the Museum of Fine Arts, Boston. [Vidani 2020]

Looking back, in the age of BIM, at an architectural or engineering drawing from a century ago, you are likely to be struck by how little information it seems to carry. The sometimes beautiful and painstakingly rendered images themselves may be elaborate, with detail that would rank high in Shannon entropy as measured by an image compression algorithm, for instance, but in terms of providing instructions to put a building together much often seems to be left out. It seems a simpler time, without the raft of large-scale details and data, sections and specifications that we might now expect, yet how could one even consider construction of something as complicated as a contemporary building without the wealth of information these provide? We are fortunate, now, to have the tools to model, represent and track every element of a design in every aspect: its geometry, material, cost, origin, construction sequence and changing condition over time. We are fortunate to be able to generate and update documents with ease. With the help of computation, designers have become the skilled coordinators of vast amounts of information that allow them to build more complex, more economical, more environmentally sensitive technological marvels than ever before.

But that is not really what DC I/O is about. Part of the reason the historic drawing appears so minimal is that a great deal of information is just not required on paper, because it is embedded in the minds and hands of those doing the construction, and in longstanding traditions that are known to all. Much of the "information" in a neoclassical stone façade is 
better left to the mason, and the joiner knows the details of window sash construction better than the drawing can convey. All of this information-vast amounts of information on materials, techniques, forms and technologies - is invisible when we look at the drawing in isolation just because it isn't all in the drawing. It is shared quite differently, communicated between masters and apprentices and between practitioners, in the community responsible for making the building what it will be. This is still the case today, only the invisible practices now include artificial intelligence and augmented reality, digital manufacturing and digital twins, blockchain and biotechnology, telecommunication and optimisation. These are the inner workings of the apparent "black box" of design computation.

DC I/O aims to be a venue for us to communicate and discuss these crucial activities of design that don't appear explicitly in the drawing, or the BIM model, or the media coverage. Some, like climate change, biotechnology and data visualisation, are not the traditional responsibilities of the designer but have their own domains in separate branches of science or technology, discussed in academic conferences far removed from practice. Others, like the increasing use of optimisation, machine learning and similar algorithms within design, are pursued within the offices of architects and engineers whose output is the completed design, and so remain well in the background of the final product. The individual innovations that occur in these contexts are some of the main drivers of progress in design; they allow us, quite literally, to build a better world. But this progress is slow when these are not shared.

The digital nature of many of these innovations means they lend themselves well to being shared. Code and data, as digital objects, can be copied and distributed to anyone instantly. This is the "information" part of the work. The papers presented here have been formatted so as to highlight this explicitly, with the second section of each paper dedicated to presenting these details as clearly as possible, sometimes in the form of an algorithm expressed in pseudo-code or sometimes in the form of data collected from a scientific experiment. It is our hope that this kind of presentation will provide a better record of the essential work in design computation that might otherwise be overlooked, both in the focus on the final form of the design and in the emphasis on the results of a traditional academic paper. And if this record is good, we should hope to see more of it in the future.

More than this, though, we should hope to innovate. The more substantial aim is not to build incrementally on the well-kept record of technological success, but to make creative leaps. Both science and design have been included here, and they are fields that differ substantially in their aims. The differences between describing the world "as it is" and the world "as it might be" are evident enough. Where a scientific experiment must often isolate the single phenomenon it seeks to explain, a design must withstand criticism on all fronts, in all its complexity. And, while a scientific discovery applies broadly, perhaps across the universe, a design is unique, for a particular client, place and time. What is less evident is how similar are the two practices. We might lose sight, if we focus on the work of famous designers or on established theories, or in the emphasis on the information and data that is the material of the computational approach, of the fact that both science and design are fundamentally social activities. An experiment must be repeatable so that other scientists can test it independently; they will either agree or disagree. A design in progress is criticised so that other designers might find its flaws; they will either agree or disagree. It is only by means of these disagreements, well-structured and targeted, that innovation happens. They are the motivators of creativity.

Such creative disagreement, for now, is not something for the computer to do. One of the greatest strengths of computation has been in standardising the channels of communication to transmit data more effectively, but innovation often needs to violate standards. Artificial intelligence offers a possible alternative, but for the moment its biggest successes remain in pattern recognition of the known rather than discovery of the novel; the algorithms that suggest the news and books we read draw on our previous likes, and appear only to reinforce what we know. If we disagree from within our isolated bubbles this does not lead to creative change. The diverse background of the design computation community is a strength inasmuch as it provides the different points of view needed to provoke the disagreements that lead to innovation.

This is the reason for the two-part structure of the papers in this volume, which we believe is a novel format and so an experiment in itself. By explicitly highlighting the "implementation" details in part two of each paper, we might hope to be able to see better the craft of our colleagues, in the form of code, data and techniques. These are the details that too often remain invisible, inside the black box. Part one of the paper, and the DC I/O conference, are intended to form the basis for the discussion that needs to be had. This is done with the recognition that our community of practice is ill suited to protecting knowledge as though between master and apprentice, but will thrive by finding better opportunities to discuss, disagree, debate, and thereby push it forward. It is a community not of tradition, but of innovation.

\section{REFERENCES}

Vidani, P. 2020. ARCHI/MAPS. ARCHI/MAPS. https://archimaps.tumblr.com/post/63598295141/construction-plandetails-for-the-museum-of-fine. 